\title{
Heterogeneity in the links between sleep arousals, amyloid- $\beta$, and cognition
}

\author{
Daphne O. Chylinski, ${ }^{1}$ Maxime Van Egroo, ${ }^{1}$ Justinas Narbutas, ${ }^{1,2}$ Martin Grignard, ${ }^{1}$ \\ Ekaterina Koshmanova, ${ }^{1}$ Christian Berthomier, ${ }^{3}$ Pierre Berthomier, ${ }^{3}$ Marie Brandewinder, ${ }^{3}$ \\ Eric Salmon, ${ }^{1,2,4}$ Mohamed Ali Bahri, ${ }^{1}$ Christine Bastin,, ${ }^{1,2}$ Fabienne Collette, ${ }^{1,2}$ Christophe Phillips, ${ }^{1,5}$ \\ Pierre Maquet, ${ }^{1,4}$ Vincenzo Muto, ${ }^{1}$ and Gilles Vandewalle ${ }^{1}$ \\ 'GIGA-Cyclotron Research Centre-In Vivo Imaging and 'Psychology and Cognitive Neuroscience Research Unit, University of \\ Liege, Liege, Belgium. ${ }^{3}$ Physip SA, Paris, France. ${ }^{4}$ Department of Neurology, University Hospital of Liege, Liege, Belgium. \\ ${ }^{5}$ GIGA-In Silico Medicine, University of Liege, Liege, Belgium.
}

BACKGROUND. Tight relationships between sleep quality, cognition, and amyloid- $\beta$ (A $\beta$ ) accumulation, a hallmark of Alzheimer's disease (AD) neuropathology, have been shown. Sleep arousals become more prevalent with aging and are considered to reflect poorer sleep quality. However, heterogeneity in arousals has been suggested while their associations with $A \beta$ and cognition are not established.

METHODS. We recorded undisturbed night-time sleep with EEC in 101 healthy individuals aged 50-70 years, devoid of cognitive and sleep disorders. We classified spontaneous arousals according to their association with muscular tone increase $(\mathrm{M}+/ \mathrm{M}-)$ and sleep stage transition ( $\mathrm{T}+/ \mathrm{T}-)$. We assessed cortical $A \beta$ burden over earliest affected regions via PET imaging and assessed cognition via neuropsychological testing.

RESULTS. Arousal types differed in their oscillatory composition in $\theta(4-8 \mathrm{~Hz})$ and $\beta(16-30 \mathrm{~Hz})$ EEG bands. Furthermore, $T+M-$ arousals, interrupting sleep continuity, were positively linked to A $\beta$ burden $\left(P=0.0053, R_{\beta^{*}}^{2}=0.08\right)$. By contrast, more prevalent $\mathrm{T}-\mathrm{M}+$ arousals, upholding sleep continuity, were associated with lower $\mathrm{A} \beta$ burden $\left(P=0.0003, R_{\beta^{*}}{ }^{*}=0.13\right)$, and better cognition, particularly over the attentional domain $\left(P<0.05, R_{\beta^{*}}^{2} \geq 0.04\right)$.

CONCLUSION. Contrasting with what is commonly accepted, we provide empirical evidence that arousals are diverse and differently associated with early AD-related neuropathology and cognition. This suggests that sleep arousals, and their coalescence with other brain oscillations during sleep, may actively contribute to the beneficial functions of sleep and constitute markers of favorable brain and cognitive health trajectories.

are co-first authors

Conflict of interest: C. Berthomier, $\mathrm{PB}$, and MB have ownership/ directorship and are employees of Physip, which owns Aseega. [18F] Flutemetamol doses were provided and cost covered by GE Healthcare Ltd. as part of an investigator sponsored study (ISS290) agreement.

Copyright: ( 2021 , Chylinski et al. This is an open access article published under the terms of the Creative Commons Attribution 4.0 International License.

Submitted: July 2, 2021

Accepted: November 10, 2021 Published: November 16, 2021

Reference information: /CI Insight. 2021;6(24):e152858. https://doi.org/10.1172/jci. insight.152858.

FUNDING. FRS-FNRS Belgium (FRSM 3.4516.11), Actions de Recherche Concertées Fédération Wallonie-Bruxelles (SLEEPDEM 17/27-09), ULiège, and European Regional Development Fund (Radiomed Project).

\section{Introduction}

Sleep is central to health and cognition, and it deteriorates with aging (1). In addition, sleep disruption is associated with Alzheimer's disease (AD), as assessed by hyperphosphorylated tau and amyloid- $\beta$ (A $\beta$ ) brain accumulation, most likely in a bidirectional manner $(2,3)$. Poorer sleep quality (4), daytime sleepiness $(5)$, reduced slow-wave sleep $(6,7)$, and sleep deprivation $(8,9)$ have been linked to higher $A \beta$ levels, but they have also been linked to poorer cognitive performance $(7,10)$.

Sleep arousals, defined as transient accelerations in sleep electroencephalogram (EEG) rhythms, are usually considered as brain reactions to internal (e.g., apnea) or external (e.g., auditory stimulus) perturbations (11). Although they are key elements of sleep microstructure, they can also shape its macrostructure and lead to a shallower sleep stage (12). Arousals are most often considered as markers of sleep disruption, thereby a detrimental and harmful sleep feature. Several conceptual definitions classified them 
almost exclusively in the context of sleep disorders (e.g., sleep disordered breathing [SDB] and, to a smaller extent, periodic limb movements syndrome [PLMS]) or, in experimental protocols, inducing arousals through external - mainly auditory - stimulation $(13,14)$. These types of studies yielded mixed results. A negative link between arousal prevalence during sleep and cognitive performance was revealed in SDB, particularly in attention, and sometimes in the executive and memory domains (15). By contrast, other investigations did not find such a relationship and imputed alterations in cognition in SDB to brain hypoxia (see ref. 15 for review). In individuals devoid of sleep pathologies, arousals evoked by auditory stimuli were reported to impact subsequent daytime alertness (16). In addition, sleep fragmentation induced by auditory stimulation is associated with higher A $\beta$ cerebrospinal fluid (CSF) content the following day (6).

Importantly, spontaneous arousals - i.e., not elicited by any identifiable internal or external stimuli also constitute an authentic element of undisturbed sleep in healthy individuals. Their mechanisms, cerebral correlates, and functional consequences remain largely unknown (11), with some authors suggesting that there may be physiologic and pathologic arousals. Understanding their respective roles might shed light on the adaptive properties of the sleeping brain and provide insight into pathological mechanisms associated with sleep disturbances (11).

Here, we assessed whether different types of spontaneous arousals during sleep were differentially associated with $\mathrm{A} \beta$ cortical deposition and cognitive performance in a cohort of healthy individuals in late midlife. We were able to tease apart different types of arousals, based on their temporal relationships with increased muscular tone and sleep stage transitions. In line with the hypothesis that arousals perturb sleep, we anticipated that arousals fragmenting sleep structure would be associated with both worse cognitive performance and $A \beta$ deposition in brain areas that are first affected by this $\mathrm{AD}$-related neuropathological process.

\section{Results}

EEG oscillations differ across arousal types. We recorded undisturbed sleep at habitual sleep times under EEG in 101 healthy individuals aged 50-70 years ( $59 \pm 5$ years; 68 women), following 1 week of regular sleep-wake schedule (Figure 1). In order to evaluate the potential heterogeneity of arousals, we split them according to 2 criteria, which we considered as relevant in research settings, as well as in clinical practice. We first chose to focus on whether arousals did trigger a sleep stage transition $(\mathrm{T}+$ ) (when they occurred within 15 seconds [15s] of a stage change) or not (T-), as arousals may or may not lead to a lighter sleep stage (12) and they have already been investigated in that regard (17). Secondly, we considered their salience, reflected by the concomitant increase in electromyogram (EMG) tone $(\mathrm{M}+)$ or its absence $(\mathrm{M}-)$, as it is among the arousal scoring criteria (needed to score an arousal in rapid eye movement [REM] sleep).

In a first step, we assessed whether characterizing sleep arousals by their association with sleep stage transition ( $\mathrm{T}+$ or $\mathrm{T}-$ ), and the cooccurrence of an EMG tone increase $(\mathrm{M}+)$ or not $(\mathrm{M}-)$ resulted in differences in their oscillatory properties. We computed individual relative power in the different EEG frequency bands defining an arousal $(\theta, 4.5-7.5 \mathrm{~Hz} ; \alpha, 8.5-11.5 \mathrm{~Hz}$; and $\beta, 16.5-29.5 \mathrm{~Hz})$. A generalized linear mixed model (GLMM) with relative power as a dependent variable (and adjusted for data distribution) first indicated that relative power changed across frequency bands $\left(\mathrm{F}_{2,294.1}=403.84\right.$, $P<0.0001$, semipartial $\left.R^{2}\left[R_{\beta^{*}}^{2}\right]=0.73\right)$. More importantly, it yielded a triple interaction between transition, EMG status, and frequency band $\left(\mathrm{F}_{2,880}=3.39, P=0.034, R_{\beta^{*}}=0.008\right)$, implying that arousals differ in their spectral composition based on the presence or absence of EMG changes and sleep stage transition (Figure 2A). This was further reflected in main effects of EMG status $\left(\mathrm{F}_{1,989.9}=75.97, P=\right.$ $\left.0.0001, R_{\beta^{*}}^{2}=0.07\right)$ and transition $\left(\mathrm{F}_{1,708.6}=39.17, P<0.0001, R_{\beta^{*}}^{2}=0.05\right)$, as well as in interactions between transition and frequency band $\left(\mathrm{F}_{2,709.5}=34.62, P<0.0001, R_{\beta^{*}}=0.09\right)$, between EMG status and frequency band $\left(\mathrm{F}_{2,979,2}=187.39, P<0.0001, R_{\beta^{*}}^{2}=0.28\right)$, and between EMG status and transition $\left(\mathrm{F}_{1,879.3}=22.55, P<0.0001, R_{\beta^{*}}=0.025\right)$. Based on this first analysis, we therefore concluded that the factors of arousal heterogeneity eloquently define 4 types of arousals $(\mathrm{T}+\mathrm{M}+, \mathrm{T}+\mathrm{M}-, \mathrm{T}-\mathrm{M}+$, and $\mathrm{T}-\mathrm{M}-$ ). We finally note that multiple post hoc comparisons within each band yielded significant differences across arousal types over the $\theta$ and $\beta$ bands (see Table 1).

Arousal heterogeneity reflects different associations with $A \beta$ burden. In line with our goal to consider very early $\mathrm{AD}$-related neuropathological process, $\mathrm{A} \beta$ burden was quantified over the regions previously reported as the earliest cortical aggregation sites (18) - the frontal medial cortex and basal part of temporal lobe (fusiform and inferior temporal gyri) in all but 1 participant. We tested in a GLMM whether associations between arousal density varied with transition $(\mathrm{T}+, \mathrm{T}-)$ and $\mathrm{EMG}(\mathrm{M}+, \mathrm{M}-)$ statuses and were associated with $\mathrm{A} \beta$ 


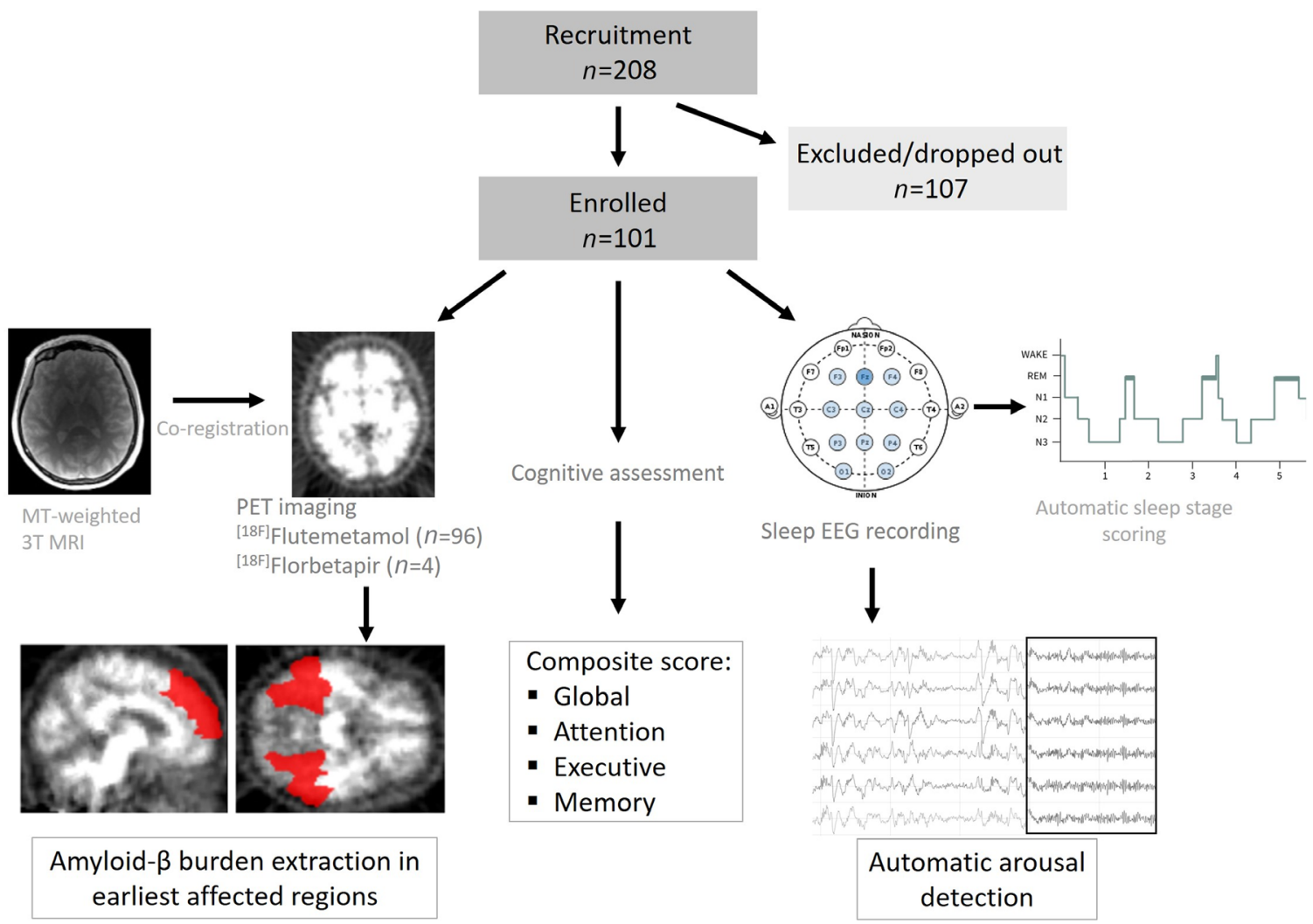

Figure 1. Overview of the study. In total, 208 participants were recruited, of which 107 did not participate in the study, as they were excluded based on inclusion criteria (see Methods), if sleep apnea were detected $\left(>15 /\right.$ hour) or decided to withdraw. Participants underwent $\left[{ }^{18} \mathrm{~F}\right] \mathrm{Flutemetamol}(n=96) /\left[{ }^{18} \mathrm{~F}\right]$ Florbetapir $(n=4)$ PET scan to assess A $\beta$ burden, which we extracted over the earliest affected regions; they were also tested via an extensive battery of neuropsychological tasks from which we extracted global score, as well as performance over 3 main cognitive domains (attention, executive, and memory); and habitual sleep was recorded via EEG from which arousals were automatically detected.

burden, while regressing out age and sex effects. We first observed a main effect of transition $\left(\mathrm{F}_{1,196}=607.52\right.$, $\left.P<0.0001, R_{\beta^{*}}^{2}=0.76\right)$ and EMG status $\left(\mathrm{F}_{1,98}=70.63, P<0.0001, R_{\beta^{*}}^{2}=0.42\right)$, as well as an interaction between EMG status and transition $\left(\mathrm{F}_{1,196}=102.32, P<0.0001, R_{\beta^{*}}=0.34\right)$, indicating that density of arousal types significantly varied. Interestingly, we did not find any significant main effect of early cortical A $\beta$ burden $\left(\mathrm{F}_{1,96}=0.26, P=0.61\right)$, age $\left(\mathrm{F}_{1,96}=2.44, P=0.12\right)$, or sex $\left(\mathrm{F}_{1,96}=0.12, P=0.73\right)$. Critically, the GLMM yielded a significant triple interaction between early cortical A $\beta$ burden, EMG status, and transition $\left(\mathrm{F}_{1,196}=7.16, P=0.008, R_{\beta^{*}}=0.035\right)$, implying that the association between arousals and early cortical $\mathrm{A} \beta$ burden depends on the concomitant change in muscular tone and sleep stage transition. The heterogeneity in spontaneous arousals was further reflected by the significant interactions between early cortical A $\beta$ burden and EMG status $\left(\mathrm{F}_{1,98}=8.64, P=0.004, R_{\beta^{*}}=0.08\right)$. Figure 3 decomposes the associations between each of the 4 types of arousals and early cortical $\mathrm{A} \beta$ burden.

We further computed a GLMM with early cortical A $\beta$ burden as a dependent variable to explore whether its association with $\mathrm{T}-\mathrm{M}+$ and $\mathrm{T}+\mathrm{M}-$ arousal truly differed in the part of $\mathrm{A} \beta$ burden variance $\mathrm{T}-\mathrm{M}+$ and $\mathrm{T}+\mathrm{M}-$ arousals explained in a more complex model, regressing out age and sex. Both associations were significant with a negative link between $\mathrm{A} \beta$ and $\mathrm{T}-\mathrm{M}+$ arousals $\left(\mathrm{F}_{1,95}=14.15, P=\right.$ $\left.0.0003, R_{\beta^{*}}^{2}=0.13\right)$ and with a positive association between $\mathrm{A} \beta$ and $\mathrm{T}+\mathrm{M}-\operatorname{arousals}\left(\mathrm{F}_{1,95}=8.16, P=\right.$ $\left.0.0053, R_{\beta^{*}}^{2}=0.08\right)$ - together with an expected main effect of age $\left(\mathrm{F}_{1,95}=13.02, P=0.0005, R_{\beta^{*}}^{2}=\right.$ $0.12)(19)$ and no main effect of $\operatorname{sex}\left(\mathrm{F}_{1}{ }_{9},=2.54, P=0.11\right)$. Critically, a post hoc contrast showed that the links between the 2 types of arousals and early cortical A $\beta$ burden were significantly different $\left(\mathrm{t}_{93}\right.$ $=3.73, P=0.0003$ ). In addition, $\mathrm{T}-\mathrm{M}+$ and $\mathrm{T}+\mathrm{M}-$ arousals were not correlated (Figure $2 \mathrm{~B}$ ). Supplementary analysis showed the same statistical picture in a GLMM, including all 4 arousal types together, with a significant post hoc contrast when considering $\mathrm{T}-\mathrm{M}+$ and $\mathrm{T}+\mathrm{M}-$ arousals versus early cortical A $\beta$ burden (Table 2). Results were not driven by arousals occurring in non-REM (NREM) or REM 
A

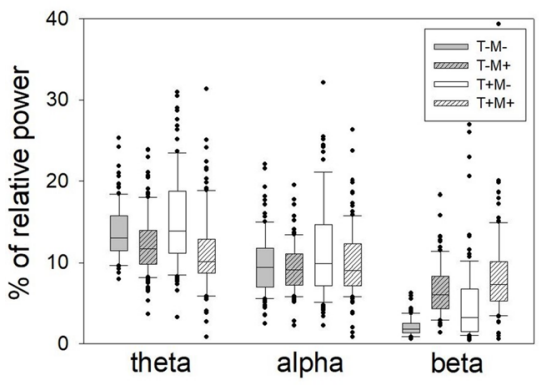

B

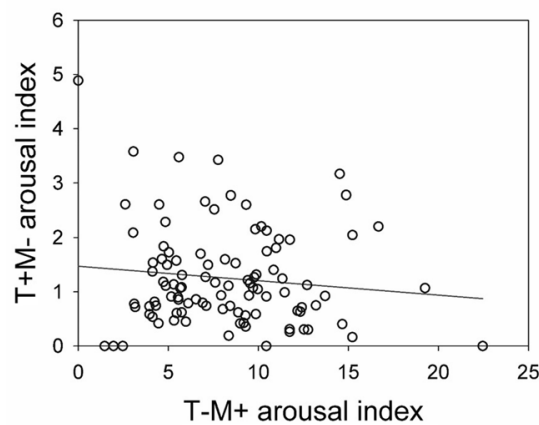

Figure 2. Spectral composition of arousal types. (A) Box plot of relative power in the $\theta(4.5-7.5 \mathrm{~Hz}), \alpha(8.5-11.5 \mathrm{~Hz})$, and $\beta(16.5-29.5 \mathrm{~Hz})$ band for T-M-, T-M+, $T+M-$, and $T+M+$ arousals with error bars. The boxes' central lines indicate the median of power values, with the bottom and upper edges showing the 25 th and 75th percentiles, respectively. $T$, arousal associated $(T+)$ or not ( $T-$ ) with sleep stage transition; $M$, arousal associated ( $M+)$ or not ( $M-)$ with an increase in EMG tone. Indexes correspond to hourly prevalence. (B) Absence of significant correlation between T-M+ arousals and $T+M-$ arousals (Spearman's $r=-0.05 ; P=0.60$ )

sleep as statistical outputs were the same if we considered the 4 types of arousals in NREM/REM separately (M+ only in REM as arousal definition is REM requires change in muscle tone) (Table 2).

Arousals linked with better $A \beta$ status are associated with better cognitive performance. We then tested whether cognition, as assessed in a global index through an extensive neuropsychological test battery, was differentially associated with the 2 arousal types showing opposite association with early cortical A $\beta$ burden. In a GLMM, the association between global cognition and $\mathrm{T}-\mathrm{M}+$ arousal index was found to be significant $\left(P=0.048, R_{\beta^{*}}^{2}=0.04\right)$, on top of the education effect, but no relation with $\mathrm{T}+\mathrm{M}-$ arousal index $(P=0.25)$, age, or sex (Table 3 and Figure 4$)$ was found. Additional exploratory GLMMs with each specific cognitive domain, in turn, showed that this association was driven by the attentional domain $\left(P=0.032, R_{\beta^{*}}^{2}=0.047\right)$ and was not significant for the executive $(P=0.09)$ or memory domains $(P=0.91)$.

We assessed the specificity of the findings for $\mathrm{T}-\mathrm{M}+$ arousals and considered the potential link between the number of full awakenings during sleep and wake after sleep onset (WASO) and the different cognitive measures in separate exploratory GLMMs. We found no link between cognition and the number of awakenings (Figure 5, A-D), while a significant negative association was detected between WASO and global cognition $\left(\mathrm{F}_{1,95}=4.66, P=0.03\right)$ which was driven by the executive domain $\left(\mathrm{F}_{1,95}=7.58, P=.007\right)$ (Figure 5, E-H). Furthermore, neither WASO nor number of awakening was associated with early cortical $\mathrm{A} \beta$ burden (Figure 5, I and J).

\section{Discussion}

Brain dynamics that buttress cerebral functions entail stationary and nonstationary interactions between neuronal populations (20). Sleep stages, which can be seen as enduring and widespread oscillatory modes

\section{Table 1. Post hoc comparisons of the relative power for each arousal type within each frequency band}

\begin{tabular}{|c|c|c|c|c|c|c|c|c|c|}
\hline \multicolumn{2}{|c|}{ Arousal type } & \multirow{2}{*}{\multicolumn{2}{|c|}{ versus arousal type }} & \multicolumn{6}{|c|}{ Frequency band relative power } \\
\hline & & & & \multicolumn{2}{|c|}{$\theta$} & \multicolumn{2}{|c|}{$\alpha$} & \multicolumn{2}{|c|}{$\beta$} \\
\hline M & $\mathrm{T}$ & M & $\mathrm{T}$ & $t$ value & Adj $P$ & $t$ value & Adj $P$ & $t$ value & $\operatorname{Adj} P$ \\
\hline- & - & - & + & -1.03 & 0.73 & -2.02 & 0.18 & -10.26 & $<0.0001$ \\
\hline- & - & + & - & 1.99 & 0.19 & 0.01 & 1.00 & -18.92 & $<0.0001$ \\
\hline- & - & + & + & 4.08 & 0.0003 & -0.35 & 0.99 & -20.91 & $<0.0001$ \\
\hline- & + & + & - & 3.30 & 0.006 & 2.02 & 0.18 & -11.41 & $<0.0001$ \\
\hline- & + & + & + & 5.11 & $<0.0001$ & 1.39 & 0.51 & -12.73 & $<0.0001$ \\
\hline+ & - & + & + & 2.61 & 0.005 & -0.43 & 0.97 & -3.29 & \\
\hline
\end{tabular}

$\mathrm{T}$, arousal associated $\left(\mathrm{T}_{+}\right)$or not $(\mathrm{T}-)$ with sleep stage transition; $\mathrm{M}$, arousal associated $\left(\mathrm{M}_{+}\right)$or not $(\mathrm{M}-)$ with an increase in EMC tone. Indexes correspond to hourly prevalence. The $t$ values were obtained from Tukey's simultaneous tests for differences of means. 
A

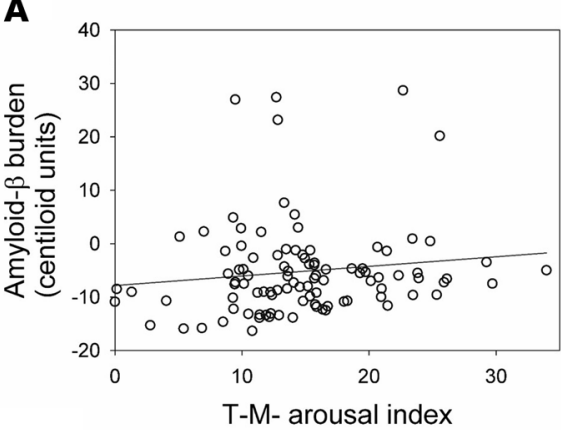

C

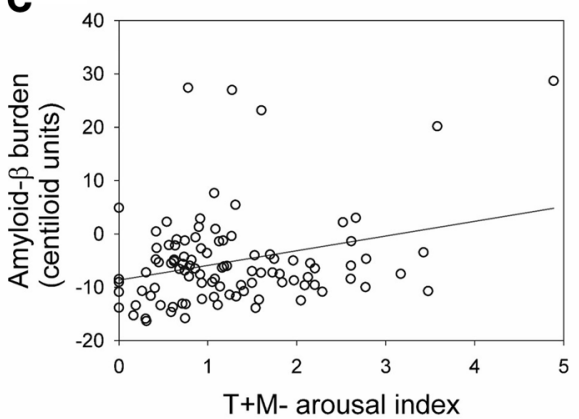

B
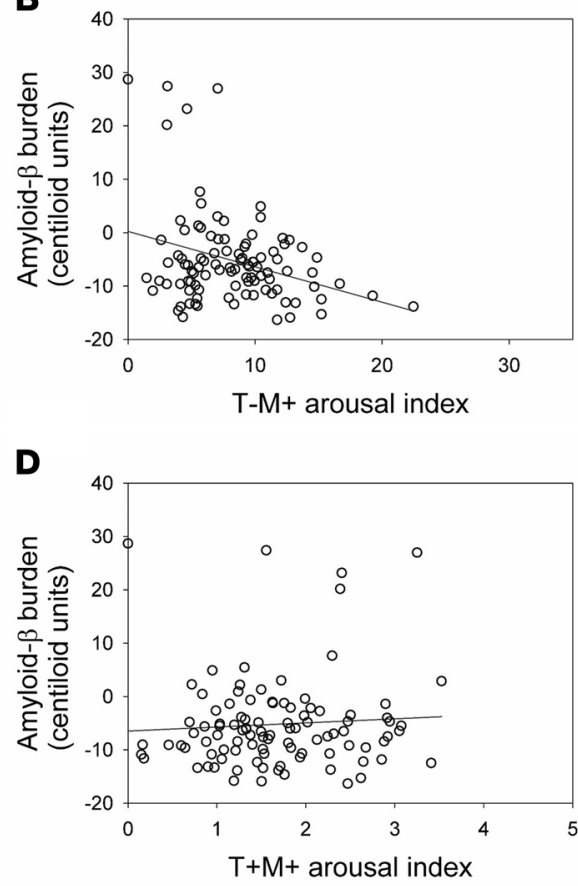

Figure 3. Associations between prevalence of different types of arousals and early cortical A $\beta$ burden. (A) No correlation between T-M- arousals and $A \beta$ burden (Spearman's $r=0.16$; $P=0.11$ ); (B) significant negative correlation between T-M+ arousals and $A \beta$ burden (Spearman's $r=-0.16, P=0.11$ ); (C) significant positive correlation between $T+M$ - arousals and $A \beta$ burden (Spearman's $r=0.17, P=0.08$ ); and (D) no correlation between $T+M+$ arousals (Spearman's $r=0.07, P=0.46$ ). See

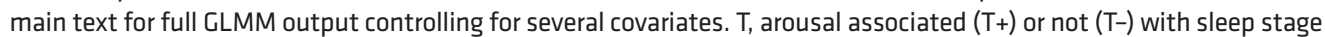
transition; $\mathrm{M}$, arousal associated $(\mathrm{M}+)$ or not $(\mathrm{M}-)$ with an increase in EMG tone. Indexes correspond to hourly prevalence.

sculpting brain activity, allow recurrent, brief, fast oscillatory activity, which sometimes leads to stage transitions $(21,22)$. Here, we focused on spontaneous arousals because their functional correlates remain undetermined. They are usually considered to induce sleep disruption and its detrimental functional consequences. However, spontaneous sleep arousals - i.e., not elicited by identifiable event - might also carry positive effects on brain functions. We quantified the prevalence of spontaneous arousals during undisturbed sleep in healthy individuals in late midlife and assessed whether it was associated with early cortical A $\beta$ deposition and cognitive performance. Based on the theoretical concept that sleep arousals are diverse (11), we classified them according to their temporal association with a change in muscular tone and a sleep stage transition. These criteria were deemed clinically relevant, as arousals may or may not affect sleep macrostructure while muscular tone constitutes an arousal marker in REM. Based on this straightforward phenotyping in a large data sample, we provide the first empirical evidence that different types of sleep arousals have distinct correlates in terms of cognition and brain amyloid burden. Indeed, we found that arousals associated with sleep transitions $(\mathrm{T}+\mathrm{M}-)$ are associated with higher cortical $\mathrm{A} \beta$ deposition in brain regions affected early on by $\mathrm{AD}$ neuropathology, suggesting their association with sleep fragmentation and worse brain status. By contrast, the more prevalent $\mathrm{T}-\mathrm{M}+$ arousals, which do not result in sleep transitions, are all the more frequent as $\mathrm{A} \beta$ deposition is low and cognitive performance superior, particularly in the attentional domain. This arousal type is therefore associated to a more favorable brain and cognitive status. Although sizes of the effects we detected remained modest, enduring small phenomenon can shape lifelong trajectories. The present findings may therefore be of particular importance since arousals have been reported to increase with age and since age represents the most important risk factor for cognitive decline and $\mathrm{AD}$ (2).

Our analyses show that the main characteristic differentiating the 2 types of arousals is whether or not they lead to a sleep stage transition. A second important criterion consisted of the concomitant increase in EMG tone. Aside from their different links with $\mathrm{A} \beta$ burden and cognition, $\mathrm{T}+\mathrm{M}-$ and $\mathrm{T}-\mathrm{M}+$ arousals are not correlated with each other and differ in their spectral composition: $\mathrm{T}+\mathrm{M}-$ bear a larger proportion of $\theta$ power, while $\mathrm{T}-\mathrm{M}+$ arousals are composed of a higher proportion of $\beta$ power. The reason $\mathrm{T}-\mathrm{M}-$ and $\mathrm{T}+\mathrm{M}+$ 
Table 2. Output of the GLMMs with A $\beta$ burden (dependent variable) and arousal types, corrected for age and sex

\begin{tabular}{|c|c|c|c|c|c|c|}
\hline \multirow[b]{3}{*}{$A \beta$ burden } & \multicolumn{6}{|c|}{ When considering arousals in all sleep stages together } \\
\hline & Age & Sex & $\mathrm{T}-\mathrm{M}-$ & $\mathrm{T}-\mathrm{M}+$ & $\mathrm{T}+\mathrm{M}-$ & $\mathrm{T}+\mathrm{M}+$ \\
\hline & $\begin{array}{c}\mathrm{F}=11.98 \\
P=0.0008 \\
R_{\beta^{*}}^{2}=0.11\end{array}$ & $\begin{array}{r}\mathrm{F}=2.11 \\
P=0.15 \\
1+\mathrm{T}+\mathrm{M}-\mathrm{C}\end{array}$ & $\begin{array}{c}\mathrm{F}=0.00 \\
P=0.99 \\
=-2.71, P=0\end{array}$ & $\begin{array}{c}\mathrm{F}=15.22 \\
P=0.0002 \\
R_{\beta^{*}}^{2}=0.14 \\
\text { djusted } P=0 .\end{array}$ & $\begin{array}{c}F=3.22 \\
P=0.076 \\
\text { timate }=-2.87\end{array}$ & $\begin{array}{l}\mathrm{F}=2.02 \\
P=0.16\end{array}$ \\
\hline & \multicolumn{6}{|c|}{ When considering arousals in NREM/REM separately } \\
\hline & Age & Sex & T-M- NREM & $T-M+N R E M$ & T+M- NREM & $T+M+N R E M$ \\
\hline$A \beta$ burden & $\begin{array}{c}\mathrm{F}=12.57 \\
P=0.0006\end{array}$ & $\begin{array}{l}\mathrm{F}=2.59 \\
P=0.11\end{array}$ & $\begin{array}{l}\mathrm{F}=0.13 \\
P=0.72\end{array}$ & $\begin{array}{c}\mathrm{F}=11.94 \\
P=0.0008\end{array}$ & $\begin{array}{l}F=3.54 \\
P=0.06\end{array}$ & $\begin{array}{l}\mathrm{F}=1.05 \\
P=0.31\end{array}$ \\
\hline & Age & Sex & & $\mathrm{T}-\mathrm{M}+\mathrm{REM}$ & & $T+M+R E M$ \\
\hline$A \beta$ burden & $\begin{array}{c}\mathrm{F}=8.55 \\
P=0.0043\end{array}$ & $\begin{array}{l}F=0.63 \\
P=0.43\end{array}$ & & $\begin{array}{l}\mathrm{F}=5.95 \\
P=0.017\end{array}$ & & $\begin{array}{l}F=0.14 \\
P=0.71\end{array}$ \\
\hline
\end{tabular}

All $\mathrm{F}$ tests had 1 (main effect) and 93 (error) degrees of freedom, except for the models with arousals in NREM/REM, which had 1 (main effect) and 95 (error) degrees of freedom. Significant associations are in bold and are accompanied by their corresponding semipartial $R^{2}\left(R_{\beta^{*}}^{2}\right)$.

arousals are not significantly associated with $\mathrm{A} \beta$ and cognition is unclear and might reside in different prevalence or in diverging effects of sleep transitions and EMG bursts, which would hinder the relationship. Future studies are warranted to further investigate this issue.

Two hypotheses can be put forward to explain the heterogeneity in arousals. On the one hand, all arousals, triggered by a common set of brain areas, might be part of a continuum in which each arousal is characterized by the intensity in its driving neural activity, its spectral composition, its associated muscular tone, and its probability of sleep stage transition. Alternatively, the 2 arousal types are distinct physiological events prompted by different triggering brain structures and propagation cerebral networks. Oddly enough, the origin of spontaneous arousals remains elusive. Recent functional MRI (fMRI) data show that subcortical regions (including the thalamus, midbrain, basal ganglia, and cerebellum) were activated during non-REM arousals, while cortical regions were deactivated (23). A recent yet-to-be-reviewed study in rodents provides evidence that arousals leading to sleep state transition are at least partly driven by the locus coeruleus (LC), brainstem source of norepinephrine, which has a strong and ubiquitous influence on distant cortical brain regions, including during sleep (24). In addition, optogenetic stimulation of the LC causes immediate sleep-to-wake transitions, from both NREM and REM sleep, and results in high-frequency EEG activity (25). Therefore, subcortical activity — for instance, in the LC - could underlie transition arousals while no-transition arousals could also merely be the reflection of

Table 3. Outputs of GLMMs assessing associations between cognitive performances (global and specific domain-dependent variables) and arousal types, while adjusting for age, sex, and education (independent variables)

\begin{tabular}{|c|c|c|c|c|c|}
\hline & $\begin{array}{l}T-M+\text { arousal } \\
\text { index }\end{array}$ & $\begin{array}{l}\mathrm{T}+\mathrm{M} \text { - arousal } \\
\text { index }\end{array}$ & Age & Sex & Education \\
\hline GLOBAL & $\begin{array}{c}\mathrm{F}=4.01 \\
P=0.048 \\
R_{\beta^{*}}^{2}=0.04\end{array}$ & $\begin{array}{l}F=1.36 \\
P=0.25\end{array}$ & $\begin{array}{c}\mathrm{F}=3.41 \\
P=0.068\end{array}$ & $\begin{array}{l}F=0.11 \\
P=0.74\end{array}$ & $\begin{array}{c}\mathrm{F}=10.92 \\
P=0.0013 \\
R_{\beta^{*}}^{2}=0.10\end{array}$ \\
\hline ATTENTION & $\begin{array}{c}\mathrm{F}=4.74 \\
P=0.032 \\
R_{\beta^{*}}^{2}=0.047\end{array}$ & $\begin{array}{l}\mathrm{F}=0.83 \\
P=0.36\end{array}$ & $\begin{aligned} \mathrm{F} & =6.08 \\
P & =0.015 \\
R_{\beta^{*}}^{2} & =0.06\end{aligned}$ & $\begin{array}{l}F=0.10 \\
P=0.75\end{array}$ & $\begin{array}{c}\mathrm{F}=4.48 \\
P=0.037 \\
R_{\beta^{*}}^{2}=0.045\end{array}$ \\
\hline MEMORY & $\begin{array}{l}\mathrm{F}=0.01 \\
P=0.91\end{array}$ & $\begin{array}{l}\mathrm{F}=0.33 \\
P=0.57\end{array}$ & $\begin{array}{l}\mathrm{F}=0.08 \\
P=0.77\end{array}$ & $\begin{array}{l}F=3.15 \\
P=0.08\end{array}$ & $\begin{array}{l}\mathrm{F}=2.35 \\
P=0.13\end{array}$ \\
\hline
\end{tabular}

All $\mathrm{F}$ tests had 1 (main effect) and 95 (error) degrees of freedom. Significant associations are in bold and are accompanied by their corresponding semipartial $R^{2}\left(R_{\beta^{*}}^{2}\right)$. T, arousal associated ( $\mathrm{T}+$ ) or not ( $\mathrm{T}-$ ) with sleep stage transition; $\mathrm{M}$, arousal associated $(\mathrm{M}+)$ or not (M-) with an increase in EMG tone. 
A

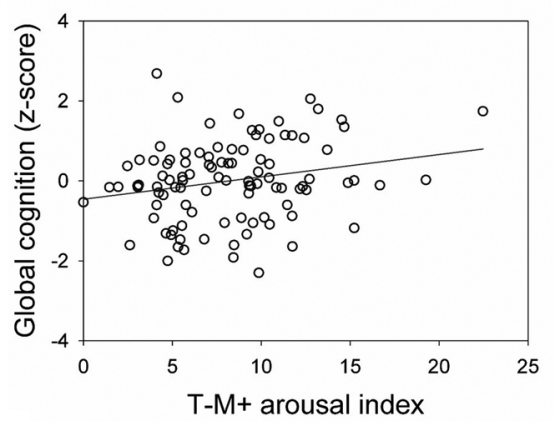

C

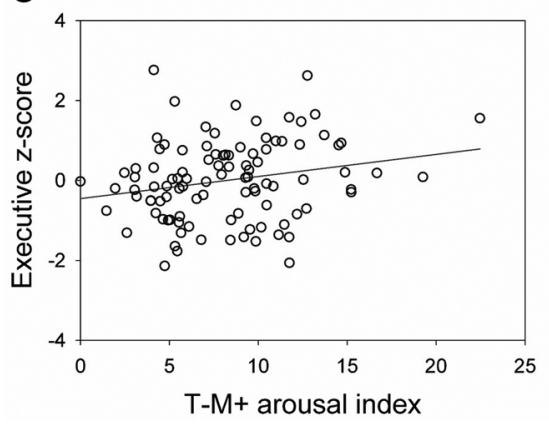

B

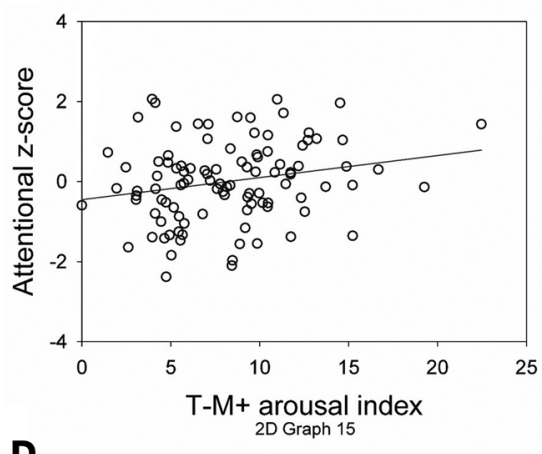

D

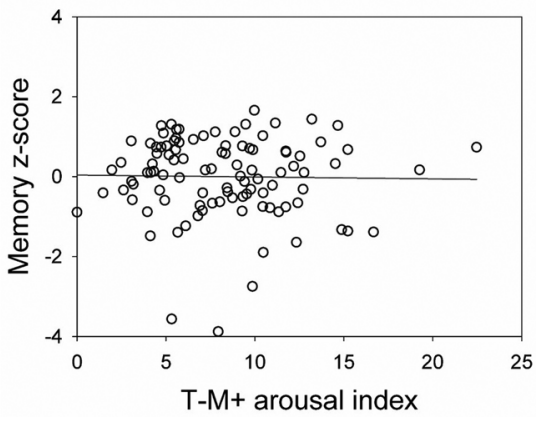

Figure 4. Association between $T-M+$ arousals prevalence and cognitive performance. (A) $T-M+$ arousals and global cognition (Spearman's $r=0.21, P=0.04$ ); (B) T-M+ arousals and attention (Spearman's $r=0.22, P=0.03$ ); (C) T-M+ arousals and executive functioning (Spearman's $r=0.21, P=0.03$ ); (D) T-M+ arousals and memory (Spearman's $r=-0.03$, $P=0.71$ ). See Table 3 for full GLMM outputs. $T$, arousal associated ( $T+$ ) or not ( $T-$ ) with sleep stage transition; $M$, arousal associated $\left(M_{+}\right)$or not $(M-)$ with an increase in EMG tone. Indexes correspond to hourly prevalence.

cortico-cortical or thalamo-cortical interplay (11). Identifying the brain sources of the 2 types of arousals would require invasive animal testing, coupling EEG to fMRI recordings in humans, or source reconstruction of high density EEG signals (22).

The cellular and molecular underpinnings of the distinct relationship between the 2 types of arousals, $\mathrm{A} \beta$ burden, and cognition are currently unknown. We can reasonably speculate that $\mathrm{T}+\mathrm{M}-$ arousals have 2 potentially deleterious impacts. Firstly, they interrupt a sleep stage and consequently all its associated cellular phenomena, like plasticity (21). Secondly, it seems possible that they considerably increase cellular activity in diffused cerebral regions, a condition conducive to increase A $\beta$ release. By contrast, one could tentatively speculate that $\mathrm{T}-\mathrm{M}+$ arousals promote $\mathrm{A} \beta$ clearance, hypothetically by increasing the pulsatility of cortical penetrating arteries (26). Additionally, $\mathrm{T}-\mathrm{M}+$ arousals might offer recurring opportunities to transiently synchronize distant brain areas, in frequency bands otherwise related to cognition during wakefulness (e.g., $\beta$ oscillations; ref. 27) without enduringly disrupting the underlying brain oscillations (i.e., sleep state), similarly to what sleep spindles allow over $\sigma$ band (12$16 \mathrm{~Hz}$ ) oscillations (28). In complex dynamics wordings, $\mathrm{T}-\mathrm{M}+$ arousals can be seen as distinct dynamics generated when the oscillatory trajectory is trapped in a local submanifold of an attractor (29), meaning the arousal would represent only a temporary breakout from the global oscillatory regime. These transient oscillations give rise to dynamic instability, despite the fact that the global manifold does not change. Dynamic instability is a form of complexity in neuronal systems that is critical for adaptive brain functions such as selection in self-organizing systems, learning, or memory (20). On the other hand, $\mathrm{T}+\mathrm{M}-$ arousals would represent a distinct type of complexity, where the involvement of the brainstem would lead to a change in oscillatory regime through a change in the attractor manifold. Similar transient oscillations have been previously reported during wakefulness and have been reported to be related to cognition (20). Further studies are needed to unravel whether higher $\mathrm{T}+\mathrm{M}-/$ lower $\mathrm{T}-\mathrm{M}+$ arousal indexes are facilitating $A \beta$ aggregation or if, conversely, accumulating $A \beta$ burden is disrupting sleep processes (2). Data in young individuals, in which current $A \beta$ detection is typically negative (18), as well as longitudinal studies are needed to address this issue. 
A

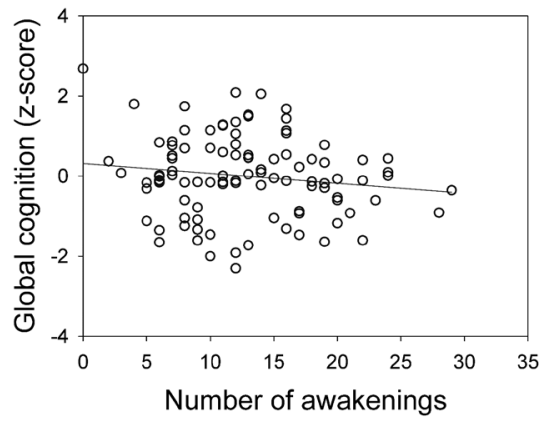

C

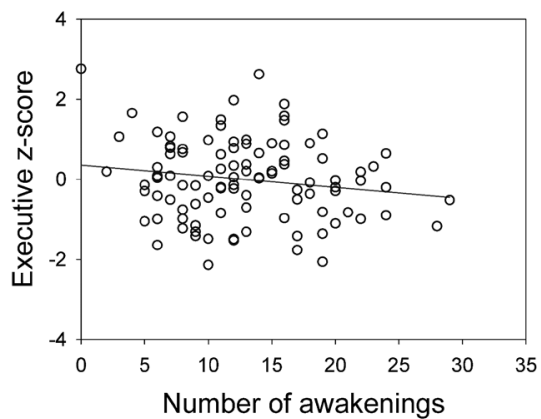

E

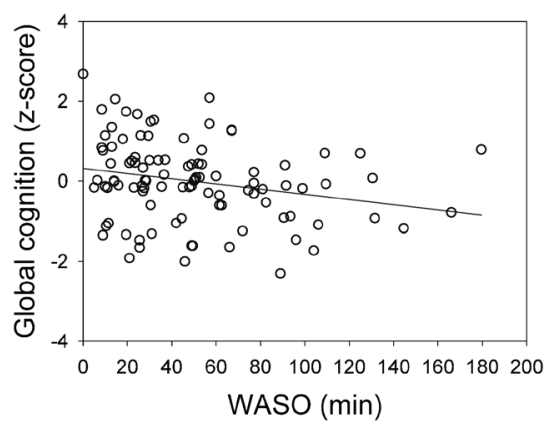

G

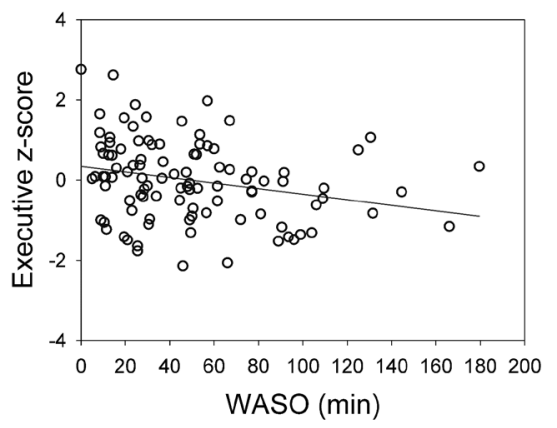

I

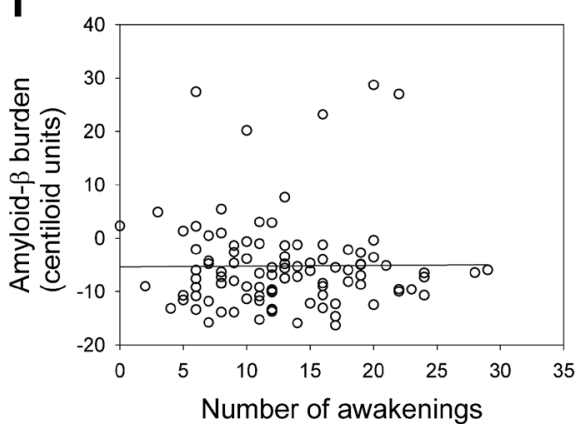

B

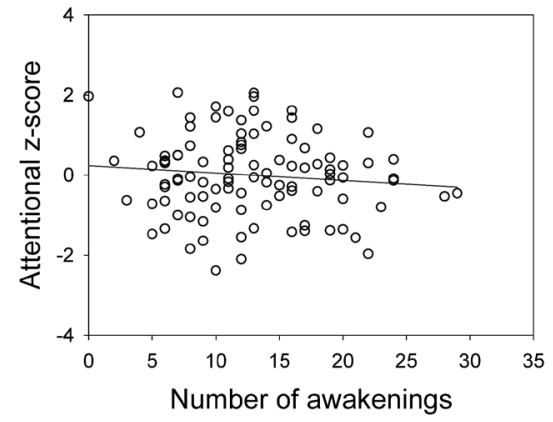

D

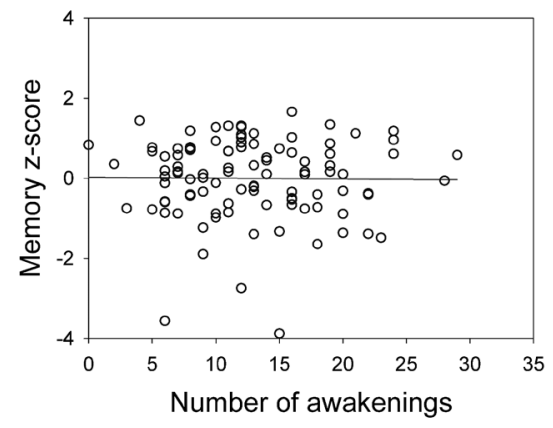

F

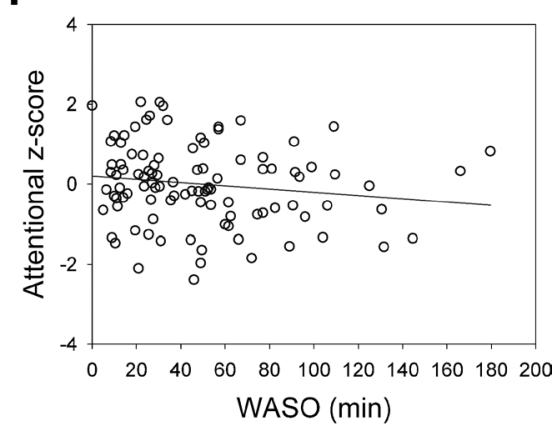

H
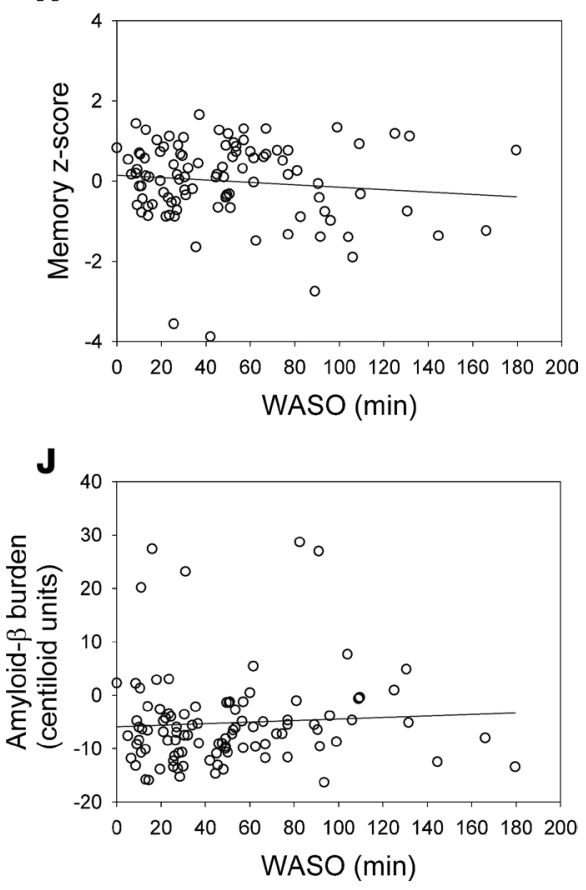

Figure 5. Association between number of awakenings and cognition. (A) Global cognitive performance (Spearman's $r=-0.10, P=$ 0.30 ; GLMM F $\left.F_{1.95}=2.21, P=0.14\right)$; (B) attentional (Spearman's $r=-0.07, P=0.48$; GLMM $F_{1,95}$ $=0.89, P=0.35$ ); (C) executive (Spearman's $r=$ $-0.11, P=0.25 ;$ GLMM F $\left._{1.95}=3.53, P=0.06\right) ;$ (D) memory performances (Spearman's $r=-0.03$, $P=0.80 ;$ GLMM F $_{1,95}=0.00, P=0.98$ ); (E) between WASO and cognition global cognitive performance (Spearman's $r=-0.24, P=0.01$; GLMM $\left.F_{1,95}=4.66, P=0.03\right)$; (F) attentional (Spearman's $r=-0.17, P=0.11$; GLMM: $F_{1,95}=$ $0.56, P=0.46$ ); (G) executive (Spearman's $r=$ $-0.28, P=0.005$;GLMM: $\mathrm{F}_{1,95}=7.58, P=0.007$ ); (H) memory performances (Spearman's $r=$ -0.06, P= 0.59; GLMM: $\mathrm{F}_{1.95}=1.11, P=0.30$ ); (I) between early cortical $A \beta$ burden and number of awakenings (Spearman's $r=0.01, P=0.90$; GLMM $\left.F_{1,95}=0.22, P=0.64\right)$; and (J) WASO (Spearman's $r=0.11, P=0.28$; GLMM $F_{1,95}=$ $0.05, P=0.83$ ). 
We emphasize that (a) our cohort only comprised healthy individuals, devoid of SDB, and (b) we focused on spontaneous arousals, which are not generated in response to detectable endogenous or exogenous perturbation (e.g., apnea or noise). Therefore, our findings probably do not apply to potentially more prevalent perturbation-induced arousals and their negative behavioral $(15,16)$ and neurodegenerative aftermaths $(6)$. We also underline that we aimed to investigate links between sleep and $A \beta$ burden early on in this neuropathological process; therefore, our volunteers did not show large $A \beta$ deposition (only 5 could be considered as $A \beta$ positive). Although $\mathrm{A} \beta$ is a hallmark of $\mathrm{AD}$ neuropathology, we do not know which volunteer will develop $\mathrm{AD}$ and, therefore, which one can be considered to undergo a true $\mathrm{AD}$ process - or an $\mathrm{AD}$-like or $\mathrm{AD}$-related process. As for any $A \beta$ signal, its predictive value remains debated. It is tantalizing to suggest, and empirically testable, that arousals found in SDB mostly consist in transition arousals, which would contribute in part to the higher risk for $\mathrm{AD}$ reported in SDB (30). We further found no significant link between early $\mathrm{A} \beta$ burden and the number of full night-time awakenings during sleep or with WASO, 2 markers related to the fragmentation of sleep macrostructure defining in part sleep quality. The associations we find with $A \beta$ burden in healthy late midlife appear, therefore, to be stronger with - if not specific to - sleep arousals, as compared with other indices of wakefulness during sleep or fragmentation of sleep. This contrast with a previous actigraphy study that reported correlations between WASO and A $\beta$ burden in participants older than those included here (mean, $76.7 \pm 3.5$ years) (31). Our findings may therefore suggest that, at a younger age ( $\sim 59$ years), the detrimental association between sleep quality and $\mathrm{AD}$ neuropathology initially concerns transition arousals, leading to sleep macrostructure fragmentation, before being subsequently detected over other markers of sleep fragmentation.

Sleep arousals may connect the sleeper's brain with the surrounding endogenous and exogenous relevant incoming information and contribute to elements of cortico-cortical information processing $(11,29)$, as done through sleep spindles, another fundamental feature of sleep microstructure (28). In other words, our findings suggest that sleep arousals, and their coalescence with other brain oscillations during sleep, may actively contribute to the beneficial functions of sleep. Arousals may interact with spindles and slow waves, however, so that we cannot rule out a contribution of these events to the effects we report. Future research should assess whether arousals are predictors of $\mathrm{A} \beta$ burden independent of other known neurophysiological elements/oscillations of sleep linked to cognition and brain health. Visual inspection of the data indicates that, despite occasional cooccurrence, slow wave arousals are not strongly nor systematically associated with any type of arousals. Our findings constitute the first empirical evidence of the conceptual existence of different arousal types differently associated to important parameters of cognitive and brain health (11). Sleep microfragmentation, as easily indexed by automatic detection of spontaneous arousals, could therefore potentially constitute a marker of favorable brain and cognitive trajectory in clinical practice, at least in late midlife adults and/or in individuals with still early AD-related neuropathology.

\section{Methods}

Study design and participants. In order to target early A $\beta$ brain deposit (18), we recruited healthy older individuals aged 50-70 years. In total, 208 volunteers were recruited, of which 101 participated in the actual study (Table $4)$. The rest were excluded due to one of the following exclusion criteria: clinical symptoms of cognitive impairment (dementia rating scale < 130; mini mental state examination $<27$ ); BMI $\leq 18$ and $\geq 29$; recent psychiatric history or severe brain trauma; documented/diagnosed sleep pathologies such as insomnia and REM behavior disorder; medication affecting the CNS; smoking; excessive alcohol ( $>14$ units/week) or caffeine ( $>5$ cups/ day) consumption; shift work in the past 6 months; or transmeridian travel in the last 2 months.

Participants were screened for sleep apnea/hypopnea syndrome during an in-lab night of sleep under polysomnography (PSG) preceding the one that was analyzed in the results section of this paper. This PSG included EEG (Fz, Cz, C3, PZ, Oz electrodes), 2 bipolar electrooculograms (EOGs), 2 bipolar submental EMG electrodes, 2 bipolar electrocardiograms (ECGs), 2 sets of bipolar leg electrodes, thorax and abdominal belts, an oximeter, a nasal canula, and a snoring sensor. As is typically done in similar sleep studies $(7,32)$, volunteers with an apnea/hypopnea index (AHI) $\geq 15 /$ hour were excluded (79 subjects had an $\mathrm{AHI} \geq 0$ and $<5$; 19 subjects had an $\mathrm{AHI} \geq 5$ and $<10$; and 3 subjects had an $\mathrm{AHI} \geq 10$ and $<15$ ). Given the low arousal index of our volunteers and the low rate of PLMS in our sample ( 9 subjects had a PLMS index $\geq 15$ ), and given that controlling for those 2 covariates did not change the statistically significant associations we found in the reported models, we did not include them in the statistical analyses reported below. One volunteer was excluded from analyses that included $\mathrm{A} \beta$ data due to corrupted PET scan data caused by technical issues during acquisition. Demographic characteristics of the study sample can be found in Table 4. 
Table 4. Sample characteristics of our data set (mean \pm SD) $N=101$.

\begin{tabular}{|c|c|}
\hline Sex & $68+/ 33 \curvearrowright$ \\
\hline Age (y) & $59.4 \pm 5.3$ \\
\hline Education (y) & $15.2 \pm 3$ \\
\hline Dementia rating scale $(\boldsymbol{N}=\mathbf{9 7})(53)$ & $142.5 \pm 1.9$ \\
\hline $\mathrm{BMI}\left(\mathrm{kg} / \mathrm{m}^{2}\right)$ & $24.6 \pm 2.9$ \\
\hline $\mathrm{AHI}(\mathrm{nb} / \mathrm{hr})$ & $3.1 \pm 2.9$ \\
\hline WASO (min) & $49.3 \pm 37.2$ \\
\hline Awakenings index (nb/hr) & $1.7 \pm 0.8$ \\
\hline$\%$ N1 & $6.2 \pm 2.7$ \\
\hline$\%$ N2 & $51.6 \pm 8.8$ \\
\hline$\%$ N3 & $19.1 \pm 6.4$ \\
\hline $\mathrm{T}-\mathrm{M}+$ index $(\mathrm{nb} / \mathrm{hr}) \pm \mathrm{SD}$ [range] & $8 \pm 4[0-22]$ \\
\hline $\mathrm{T}+$ arousal index $(\mathrm{nb} / \mathrm{hr}) \pm \mathrm{SD}$ [range] & $3 \pm 1[0-6]$ \\
\hline $\mathrm{T}+\mathrm{M}-$ index $(\mathrm{nb} / \mathrm{hr}) \pm \mathrm{SD}$ [range] & $1 \pm 1[0-5]$ \\
\hline $\mathrm{T}+\mathrm{M}+$ index $(\mathrm{nb} / \mathrm{hr}) \pm \mathrm{SD}[$ range $]$ & $2 \pm 1[0-4]$ \\
\hline $\mathrm{M}$ - arousal index $(\mathrm{nb} / \mathrm{hr}) \pm \mathrm{SD}$ [range] & $16 \pm 7[0-36]$ \\
\hline $\mathrm{M}+$ arousal index $(\mathrm{nb} / \mathrm{hr}) \pm \mathrm{SD}$ [range] & $10 \pm 4[0-24]$ \\
\hline
\end{tabular}

Sleep assessment. Participants came to the lab for an adaptation night under PSG, after which those with sleep an $\mathrm{AHI} \geq 15 /$ hour were excluded from further participation. Volunteers were required to follow a regular sleep-wake schedule ( \pm 30 minutes) for 1 week based on their preferred bed and wake-up times before sleep EEG recording, in order to record their sleep in settings as close as possible to habitual conditions. Compliance was verified using sleep diaries and wrist actigraphy (Actiwatch, Cambridge Neurotechnology). Participants then joined the laboratory about 6.5 hours prior to habitual sleep time and were maintained in dim-light thereafter. Undisturbed habitual sleep was recorded with N7000 amplifiers (EMBLA, Natus) using 11 EEG derivations placed according to the 10-20 system (F3, Fz, F4; C3, Cz, C4; P3, Pz, P4; O1, O2 electrodes), 2 bipolar EOGs, and 2 bipolar submental EMG electrodes. Recordings were sampled at $200 \mathrm{~Hz}$ and rereferenced to the mean of the 2 mastoids.

Arousal detection. Sleep stage scoring and arousal detection were carried out in separate steps by 2 independent algorithms. Sleep stage scoring was performed in 30-second windows using a validated algorithm (ASEEGA, Physip) (33, 34). Automatic arousal detection was then computed as it is objective and reproducible, and because it saves time (35). We used an individually tailored validated algorithm based on the American Academy of Sleep Medicine (AASM) definition (12) of arousal but without using sleep stage information. Automatic scorings were visually inspected following computation.

In brief, arousal detection is performed over all electrodes on whole-night recordings split into 1-second epochs in 2 successive steps computed over the power in the broad- $\alpha(7-13 \mathrm{~Hz}), \beta(16-30 \mathrm{~Hz})$, and lower- $\theta(3-7 \mathrm{~Hz})$ frequency bands, excluding the $\sigma$ band $(11-16 \mathrm{~Hz})-$ i.e., corresponding to frequency of sleep spindles - which cannot be considered as arousals. A fixed threshold is first applied to detect abnormal EEG activity relatively to the whole-night recording: any 1-second epoch with power in any of the 3 frequency bands higher than the whole-night median value in each frequency band is considered as a potential arousal. The second step adapts the threshold to account for the specific EEG background activity in a shorter time window. A specific threshold is computed for each 30-second window: all 1-second epochs without concomitant EMG tone increase are selected, as well as the first ten 1-second epochs without EMG increase before and after the 30-second window being evaluated; threshold of each 
frequency band consists in the median power over the selected 1-second epochs. Events composed of at least 3 consecutive 1 -second epochs with changes in EEG frequencies higher than twice the local median and 1 median of the whole recording for that frequency band were considered as arousals. For detailed explanations on the method, see ref. 35 .

In order to evaluate the potential heterogeneity of arousals, we split them according to 2 criteria, which we considered as relevant in research settings, as well as clinical practice. The first criterion addressed whether arousals did trigger a sleep stage transition $(\mathrm{T}+)$ (when they occurred within 15 seconds of a stage change - in the second half of an epoch preceding a stage change or in the first half of an epoch assigned a different stage than the previous epoch) or whether they did not ( $\mathrm{T}-$ ). The second criterion addressed their salience, reflected by the concomitant increase in EMG tone $(\mathrm{M}+)$ or its absence $(\mathrm{M}-)$.

Spectral analysis of arousals' power was carried out through a time-frequency analysis on the first 3 seconds of arousals using Morlet's wavelet transform in SPM12 (https://github.com/spm/spm12/, commit SPM12 r7219) on Fz electrode. Detrending was done over the $500 \mathrm{~ms}$ prior to the arousal event. Data were then averaged per arousal type prior to summing in the typical EEG bands that may compose an arousal $(\theta, 4.5-7.5 \mathrm{~Hz}$; $\alpha, 8.5-11.5 \mathrm{~Hz}$; and $\beta, 16.5-29.5 \mathrm{~Hz}$ ). Given the variety of factors that impact total power (e.g., conductivity of the involved tissues, such as scalp, skull, CSF) (36), and thus renders it complex to compare across subjects, relative power of each band was computed through a normalization relative to $0.5-30 \mathrm{~Hz}$ total power.

MRI data. MRI data were used in order to determine the region of interest used for extraction of $\mathrm{A} \beta$ burden value based on PET images. Quantitative multiparametric MRI acquisition was performed on a 3-Tesla MR scanner (Siemens MAGNETOM Prisma, Siemens Healthineers) to get a magnetization transfer-weighted (MT-weighted) contrast, based on multi-echo 3D fast low angle shot at $1 \mathrm{~mm}$ isotropic resolution (37) (with flip angle $=6^{\circ}$ and application of additional off-resonance Gaussian-shaped RF pulse). MRI multiparameter maps were processed with the hMRI toolbox (38) (http://hmri.info) and SPM12 (Welcome Trust Centre for Neuroimaging, London, United Kingdom) to obtain a quantitative MT map and segmented images (gray matter, white matter, CSF), normalized to the standard MNI space using unified segmentation (39).

PET scan. A $\beta$ PET imaging was performed using $\left[{ }^{18} \mathrm{~F}\right]$ Flutemetamol, except for 3 volunteers for which $\left[{ }^{18} \mathrm{~F}\right]$ Florbetapir was used. PET scans were performed on an ECAT EXACT+ HR scanner (Siemens). Participants received a single dose of the radioligand in the antecubital vein (target dose $185 \mathrm{MBq}$ ); images acquisition started 85 minutes after the injection and consisted of 4 frames of 5 minutes, followed by a 10 -minute transmission scan using ${ }^{68} \mathrm{Ge}$ line sources. Images were reconstructed using filtered back-projection algorithm including corrections for measured attenuation, dead time, random events, and scatter using standard software (Siemens ECAT, HR + V7.1, Siemens/CTI). Individual PET average images were produced using all frames and were then manually reoriented according to MT-weighted structural MRI volumes and coregistered to the individual space structural MT map. Flow-field deformation parameters obtained from DARTEL spatial normalization of the MT maps were applied to averaged coregistered PET images (40). We did not provide correction for partial volume effect, as this type of PET processing was not included in Centiloid scaling pipeline (41). Volumes of interest were determined using the automated anatomical labeling (AAL) atlas (42). Standardized uptake value ratio (SUVR) was computed using the whole cerebellum as a reference region (41). As images were acquired using 2 different radioligands, their SUVR values were converted into Centiloid units (41). A $\beta$ burden was averaged over a composite mask covering the previously reported earliest aggregation sites for A $\beta$ pathology (18) - frontal medial cortex and basal part of temporal lobe (fusiform and inferior temporal gyri).

Cognitive assessment. A cognitive battery of neuropsychological tasks was carried out in 2 sessions, while well rested. A first session of $\sim 1$ hour was performed in the afternoon prior to the sleep assessment, approximately 7.5 hours before habitual bedtime, and a second session of $\sim 1.5$ hours was performed on another day (between 12 and 6 hours prior to habitual bedtime). From those 2 sessions, 3 domain-specific composites scores were computed for the memory, executive function, and attentional domains, and they consisted of the standardized sum of the standardized domain-specific scores, where higher values indicate better performance. A fourth global cognitive score consisted of the standardized sum of the domain-specific composite scores.

The first session comprised (a) mnemonic similarity task (MST) (43); (b) category verbal fluency (letter and animals) (44); (c) digit symbol substitution task (DSST) (45); (d) visual N-back task (1-, 2-, and 3-back variants) (46); and (e) choice reaction time (CRT) (47). The second session of $\sim 1.5$ hours was performed on another day (between 12 and 6 hours prior to habitual bedtime) and comprised (a) direct and inverse digit span task (45); (b) free and cued selective reminding test (FCSRT) (48); (c) a computerized version of the 
Stroop test (49); (d) trail making test (TMT) (50), and (e) D2 attention test (51).The memory score consisted of the FCSRT (sum of all 4 free recalls) and the recognition memory score from the MST. The executive function score included verbal fluency tests (letter and animals score for 2 minutes), inverse order digit span, TMT (part B), N-back (3-back variant), and Stroop test (interfering item errors). The attentional score comprised the DSST, TMT (part A), N-back (1-back variant), D2 (Gz-F), and CRT (reaction time to dissimilar items).

Statistics. Statistical analyses were performed in SAS 9.4 (SAS Institute) using GLMMs. The distribution of dependent variables was determined by fitting all parametric probability distributions to data, using the "allfitdist" function in Matlab 2015 (http://amir.eng.uci.edu/MvCAT.php),_and GLMMs were adapted accordingly as preconized by SAS statisticians. A subject was treated as a random factor (intercept); each model included sex and age as covariates, as well as education for models with cognitive score as dependent variables. Statistical significance threshold was set at $P<0.05$ as no correction for multiple comparisons were required. The association between arousals and $\mathrm{A} \beta$ was tested in a single model, including arousal density as dependent variable together with transition $(\mathrm{T}+, \mathrm{T}-)$ and $\mathrm{EMG}(\mathrm{M}+, \mathrm{M}-)$ arousal statuses and early $\mathrm{A} \beta$ burden as regressors (as well as sex and age). The association between arousal and cognition was tested in a single model including global cognition score as a dependent variable together with $\mathrm{T}+\mathrm{M}-$ and $\mathrm{T}-\mathrm{M}+$ arousal density as regressors (as well as sex and age). Kenward-Roger correction was used to determine degrees of freedom. $R_{\beta^{*}}$ values were computed to estimate the effect sizes of significant fixed effects and statistical trends in all GLMMs (52). $P$ values in post hoc contrasts (difference of least square means) were adjusted for multiple testing using Tukey's procedure. Cook's distance was used to assess the potential presence of outliers driving the associations, and as values ranged below 0.4 , no data point was excluded from the analyses (a Cook's distant $>1$ is typically considered to reflect outlier value).

Study approval. The study was registered with EudraCT 2016-001436-35. All procedures were approved by the Hospital-Faculty Ethic Committee of ULiège. All participants signed an informed consent prior to participating in the study.

\section{Author contributions}

Study concept and design were contributed by ES, PM, CP, C Bastin, FC, and GV. Data acquisition, analysis, and interpretation were contributed by DOC, MVE, JN, MG, EK, C. Berthomier, PB, MB, ES, MAB, C Bastin, FC, CP, PM, VM, and GV. DOC and GV drafted the first version of the manuscript. All authors revised the manuscript and had final responsibility for the decision to submit for publication. While all co-first authors tightly collaborated to acquire and analyze the data for an equivalent time and workload, each of the co-first authors had their own aspect of the data to deal with in priority. The order reflects these priorities.

\section{Acknowledgments}

We thank M. Blanpain, P. Cardone, M. Cerasuolo, E. Lambot, P. Ghaemmaghami, C. Hagelstein, S. Laloux, E. Balteau, A. Claes, C. Degueldre, B. Herbillon, P. Hawotte, B. Lauricella, A. Lesoine, A. Luxen, X. Pepin, E. Tezel, D. Marzoli, C. Schmidt, and P. Villar González for their help in different steps of the study. This work was supported by Fonds National de la Recherche Scientifique (FRSFNRS, FRSM 3.4516.11, F.4513.17 and T.0242.19, Belgium); Fondation Recherche Alzheimer (SAOFRA 2019/0025); Actions de Recherche Concertées (ARC SLEEPDEM 17/27-09) of the Fédération Wallonie-Bruxelles, University of Liège (ULiège); Fondation Simone et Pierre Clerdent; and European Regional Development Fund (ERDF, Radiomed Project). $\left[{ }^{18} \mathrm{~F}\right]$ Flutemetamol doses were provided and cost covered by GE Healthcare Ltd. as part of an investigator-sponsored study (ISS290) agreement. This agreement had no influence on the protocol and results of the study reported here. MVE, C. Bastin, FC, CP, and GV are/were supported by the F.R.S.-FNRS Belgium. VM was supported by European Research Council starting grant (ERC-StG 757763). C. Berthomier, PB, and MB are owners of Physip, the company that analyzed the EEG data as part of a collaboration

Address correspondence to: Gilles Vandewalle, GIGA-Cyclotron Research Centre-In Vivo Imaging, Bâtiment B30, Allée du Six Août, 8, 4000 Liège, Belgium. Phone: 3243662316; gilles.vandewalle@uliege.be.

MVE's present address is: Faculty of Health, Medicine and Life Sciences, School for Mental Health and Neuroscience, Alzheimer Centre Limburg, Maastricht University, Maastricht, The Netherlands. 
1. Bassetti C, et al eds. ESRS European Sleep Medicine Textbook. European Sleep Research Society; 2014:

2. Van Egroo M, et al. Sleep-wake regulation and the hallmarks of the pathogenesis of Alzheimer's disease. Sleep. 2019;42(4):zsz017.

3. Ju YES, et al. Sleep and Alzheimer disease pathology--a bidirectional relationship. Nat Rev Neurol. 2014;10(2):115-119.

4. Spira AP, et al. Self-reported sleep and $\beta$-amyloid deposition in community-dwelling older adults. JAMA Neurol. 2013;70(12):1537-1543.

5. Carvalho DZ, et al. Association of excessive daytime sleepiness with longitudinal $\beta$-amyloid accumulation in elderly persons without dementia. JAMA Neurol. 2018;75(6):672-680.

6. Ju YS, et al. Slow wave sleep disruption increases cerebrospinal fluid amyloid- $\beta$ levels. Brain. 2017;140(8):2104-2111.

7. Mander BA, et al. $\beta$-amyloid disrupts human NREM slow waves and related hippocampus-dependent memory consolidation. Nat Neurosci. 2015;18(7):1051-1057.

8. Ooms S, et al. Effect of 1 night of total sleep deprivation on cerebrospinal fluid $\beta$-amyloid 42 in healthy middle-aged men: a randomized clinical trial. JAMA Neurol. 2014;71(8):971-977.

9. Lucey BP, et al. Effect of sleep on overnight cerebrospinal fluid amyloid $\beta$ kinetics. Ann Neurol. 2018;83(1):197-204.

10. Scullin MK, Bliwise DL. Sleep, cognition, and normal aging: integrating a half century of multidisciplinary research. Perspect Psychol Sci. 2015;10(1):97-137.

11. Halasz P, et al. The nature of arousal in sleep. J Sleep Res. 2004;13(1):1-23.

12. Iber C, et al. The AASM Manual for the Scoring of Sleep and Associated Events: Rules, Terminology and Technical Specifications. https://www.sleep.pitt.edu/wp-content/uploads/2020/03/The-AASM-Manual-for-Scoring-of-Sleep-and-AssociatedEvents-2007-.pdf. Accessed November 18, 2021.

13. Philip P, et al. Sleep fragmentation in normals: a model for sleepiness associated with upper airway resistance syndrome. Sleep. 1994;17(3):242-247.

14. Rudzik F, et al. Sleep spindle characteristics and arousability from nighttime transportation noise exposure in healthy young and older individuals. Sleep. 2018;41(7).

15. Aloia MS, et al. Neuropsychological sequelae of obstructive sleep apnea-hypopnea syndrome: a critical review. J Int Neuropsychol Soc. 2004;10(5):772-785

16. Roehrs T, et al. Experimental sleep fragmentation. Sleep. 1994;17(5):438-443.

17. Stepanski E, et al. Sleep fragmentation and daytime sleepiness. Sleep. 1984;7(1):18-26.

18. Grothe MJ, et al. In vivo staging of regional amyloid deposition. Neurology. 2017;89(20):2031-2038.

19. Jack CR, et al. Age-specific and sex-specific prevalence of cerebral $\beta$-amyloidosis, tauopathy, and neurodegeneration in cognitively unimpaired individuals aged 50-95 years: a cross-sectional study. Lancet Neurol. 2017;16(6):435-444.

20. Friston KJ. The labile brain. I. Neuronal transients and nonlinear coupling. Philos Trans R Soc Lond B Biol Sci. 2000;355(1394):215-236.

21. Tononi G, Cirelli C. Sleep and the price of plasticity: from synaptic to cellular homeostasis to memory consolidation and integgration. Neuron. 2014;49(18):1841-1850.

22. Dang-Vu TT, et al. Spontaneous neural activity during human slow wave sleep. Proc Natl Acad Sci U S A. 2008;105(39):15160-15165.

23. Zou G, et al. Functional MRI of arousals in nonrapid eye movement sleep. Sleep. 2020;43(2):zsz218.

24. Kjaerby C, et al. Dynamic fluctuations of the locus coeruleus-norepinephrine system underlie sleep state transitions [preprint]. https://doi.org/10.1101/2020.09.01.274977. Posted on bioRxiv September 2, 2020.

25. Carter M, et al. Tuning arousal with optogenetic modulation of locus coeruleus neurons. Nat Neurosci. 2010;13(12):1526-1533

26. Plog BA, Nedergaard M. The glymphatic system in central nervous system health and disease: past, present, and future. Annu Rev Pathol. 2018;13:379-394.

27. Spitzer B, Haegens S. Beyond the status quo: a role for beta oscillations in endogenous content (Re)activation. eNeuro. 2017;4(4):ENEURO.0170-17.2017.

28. Steriade M. The corticothalamic system in sleep. Front Biosci. 2003;8:d878-d899.

29. Friston KJ. The labile brain. II. Transients, complexity and selection. Philos Trans R Soc Lond B Biol Sci. 2000;355(1394):237-252.

30. Bubu OM, et al. Sleep, cognitive impairment, and Alzheimer's disease: a systematic review and meta-analysis. Sleep. 2017;40(1).

31. Ettore E, et al. Relationships between objectives sleep parameters and brain amyloid load in subjects at rist to Alzheimer's disease: the INSIGHT-preAD study. Sleep. 2019;42(9):zsz137.

32. André C, et al. Association of sleep-disordered breathing with Alzheimer disease biomarkers in community-dwelling older adults: a secondary analysis of a randomized clinical trial. JAMA Neurol. 2020;77(6):716-724.

33. Berthomier C, et al. Automatic analysis of single-channel sleep EEG: validation in healthy individuals. Sleep. 2007;30(11):1587-1595.

34. Peter-Derex L, et al. Automatic analysis of single-channel sleep EEG in a large spectrum of sleep disorders. J Clin Sleep Med. 2021;17(3):393-402.

35. Chylinski D, et al. Validation of an automatic arousal detection algorithm for whole-night sleep EEG recordings. Clocks Sleep. 2020;2(3):258-272.

36. Laarne $\mathrm{P}$, et al. Effects of tissue resistivities on electroencephalogram sensitivity distribution. Med Biol Eng Comput. 1999;37(5):555-559.

37. Weiskopf N, Helms G. Multi-parameter mapping of the human brain at $1 \mathrm{~mm}$ resolution in less than 20 minutes. Proc Int Soc Magn Reson Med. 2008;16:2241.

38. Tabelow K, et al. hMRI - a toolbox for quantitative MRI in neuroscience and clinical research. Neuroimage. 2019;194:191-210.

39. Ashburner J, Friston KJ. Unified segmentation. Neuroimage. 2005;26(3):839-851.

40. Ashburner J. A fast diffeomorphic image registration algorithm. Neuroimage. 2007;38(1):95-113.

41. Klunk WE, et al. The Centiloid Project: standardizing quantitative amyloid plaque estimation by PET. Alzheimers Dement. 2015;11(1):1-15.

42. Tzourio-Mazoyer N, et al. Automated anatomical labeling of activations in SPM using a macroscopic anatomical parcellation of the MNI MRI single-subject brain. Neuroimage. 2002;15(1):273-289.

43. Stark SM, et al. A task to assess behavioral pattern separation (BPS) in humans: Data from healthy aging and mild cognitive 
impairment. Neuropsychologia. 2013;51(12):2442-2449.

44. Cardebat D, et al. [Formal and semantic lexical evocation in normal subjects. performance and synamics of production as a function of sex, age, and education level]. Acta Neurol Belg. 1990;90:207-217.

45. Tulsky D, et al eds. The WAIS III - WMS III Technical Manual. Harcourt Brace \& Company; 1997.

46. Kirchner WK. Age differences in short-term retention of rapidly changing information. J Exp Psychol. 1958;55(4):352-358.

47. Zimmermann P, Fimm B. A test battery for attentional performance. In: Leclercq P, Zimmermann P, eds. Applied Neuropsychology of Attention: Theory, Diagnosis and Rehabilitation. Psychology Press; 2002:110-151.

48. Grober E, et al. Screening for dementia by memory testing. Neurology. 1988;38(6):900-903.

49. Stroop JR. Studies of interference in serial verbal reactions. J Exp Psychol. 1935;18(6):643-662.

50. Bowie CR, Harvey PD. Administration and interpretation of the Trail Making Test. Nat Protoc. 2006;1(5):2277-2281.

51. Brickenkamp R, et al eds. D2R - d2 Test of Attention. Hogrefe; 1966.

52. Jaeger BC, et al. An $\mathrm{R}^{2}$ statistic for fixed effects in the generalized linear mixed model. J Appl Stat. 2017;44(6):1086-1105.

53. Mattis S. Mental Status Examination for Organic Mental Syndrome in the Elderly Patients. In: Bellak L, Karasu T, eds. Geriatric Psychiatry: A Handbook for Psychiatrists and Primary Care Physicians. Grune \& Stratton; 1976:77-121 